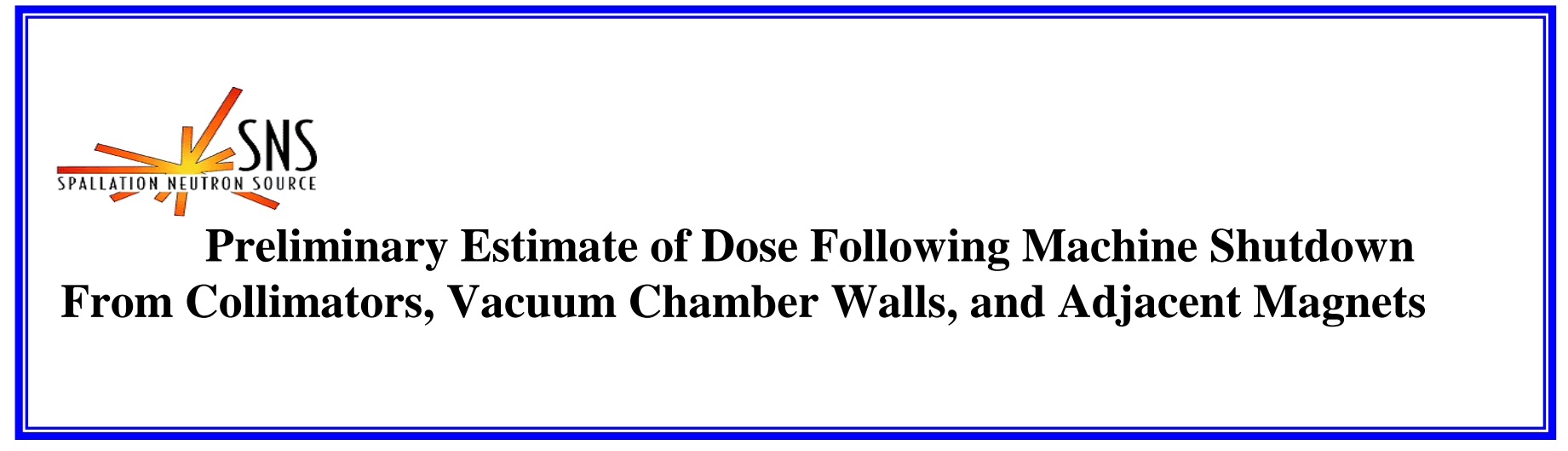

BNL/SNS TECHNICAL NOTE

NO. 067

H. Ludewig

October 18, 1999

COLLIDER ACCELERATOR DEPARTMENT BROOKHAVEN NATIONAL LABORATORY UPTON, NEW YORK 11973 


\section{PRELIMINARY ESTIMATE OF DOSE FOLLOWING MACHINE SHUTDOWN FROM COLLIMATORS, VACUUM CHAMBER WALLS, AND ADJACENT MAGNETS.}

\subsection{Introduction}

The vacuum chamber wall of the SNS are subject to an average proton loss equivalent to one Watt $/ \mathrm{m}$, while operating under normal conditions. These lost protons will cause spallation reactions in the chamber walls which will decay, and result in residual gamma-ray radiation following machine shutdown. This radiation will contribute to the back-ground radiation level in the various tunnels (HEBT, Ring, and RTBT). In addition, protons will scatter out of the vacuum chamber and interact with adjacent magnets, activating them and further adding to the tunnel background radiation level. In the case of the collimators, intentionally captured halo protons will cause spallation product generation in the collimator structure, the bulk of which will be contained by the collimator and surrounding shielding. A fraction will leak out of the collimator and also add to the gamma-ray background radiation level. In addition, there will be a strong anisotropy in the distribution of the radiation leaving the collimator. Radiation leaking out through shielding will be strongly attenuated. However, photons leaving the collimator volume in the direction of the connected vacuum chambers will be essentially uninhibited, and will be reduced only by the fact that the view angle is not optimal. This radiation source must by taken into account in maintenance operations around the collimator vacuum chamber, and connected equipment. The collimator will thus act as a "flash-light", with a beam of gamma-rays emanating from each end, and a greatly reduced source leaving from the shield surfaces. In the discussion below an estimate of the increased background dose due to machine operation, and the "flash-light" effect will be made.

Collimators are located in all the transfer line tunnels and the ring tunnel. In the HEBT tunnel two collimators are located at the end of the LINAC and one approximately half way around the tunnel. In the ring the collimators are located in the straight section after the injection straight, thus confining all the additional dose due to collimators to this section. The RTBT has two collimators located approximately half way along the line, and one at the end just before the target. The last collimator also acts as a shield for the accelerator from back-streaming radiation off the target. A schematic layout of the collimator locations is shown on Fig. 1.

\subsection{Analysis method and model}

In order to carry out the above estimate the following sequence of calculations have to be carried out:

1) Determination of the spallation product mass distribution, and the neutron source flux following exposure to the proton beam, 
2) Determination of a space dependent neutron flux within the collimator body, vacuum chamber, or magnet; required to obtain the appropriate cross sections for all the nuclides in the spallation product decay chains. The space dependence being defined by the cell structure.

3) Follow the decay chains of the spallation products using the appropriate cross sections, neutron flux level (determined by the proton flux, and thus the accelerator power level, and loss fraction), and run time, and

4) Tracking the gamma-rays resulting from the decay of the spallation products in each volume of interest to the outside surface and beyond into the tunnel.

The first step is carried out using the LAHET [1] code, with the proton beam halo confined to a cylindrical shape impacting the collimator front face. It is assumed that the proton beam inside the collimator tube passes through without interaction. This calculation results in files which store the history of all reaction products associated with the nuclear interactions due to primary protons. The second step uses the output files generated by LAHET as input to MCNP [2] which is used to determine the neutron flux in all the volumes of interest within the collimator in 64 energy groups. The number and structure of the energy groups is identical with that of the CINDER-90 [3] cross section library used for this analysis. This library contains nuclear data for approximately 3200 nuclides. The third step of the calculation is carried out using the ORIGEN2S [4] code which uses the spallation product mass distribution determined by LAHET, the cross sections determined by weighting the cross sections of the CINDER-90 library by the neutron energy spectra determined by the MCNP calculation, and operating parameters of the machine (power level, halo fraction, and run time). This step results in the gamma-ray source in each of the volumes of interest. This source is given as a function of time following shutdown of the accelerator. Finally, this spatially distributed source is used as an input to another MCNP calculation in which the gamma-rays are transported through the collimator and out into the environment surrounding the collimator. The photon flux at selected points outside the collimator is converted to dose in units of $\mathrm{rem} / \mathrm{hr}$, using the appropriate conversion factor.

An illustration of the collimator/shield combination used in this analysis is shown in Fig. 2. There are a total of 29 cells in the collimator, with the bulk of them located in the collimator tube and particle bed. The source in the coolant volumes are not included in the gamma-ray transport step, since it is assumed that the collimator will be drained during the servicing operations, or if a replacement is needed. The surrounding iron shield is $65 \mathrm{~cm}$ thick in the direction along the beam, and $20 \mathrm{~cm}$ thick in the radial direction. The proton halo impinges on a cylindrical area on the face of the collimator. In carrying out the ORIGEN-2S calculations it is assumed that the accelerator operates at $2 \mathrm{MW}$ for 180 days, with a loss fraction of 0.001 .

In the case of the vacuum chamber/magnet combination, the source was assumed to be due to protons interacting with the vacuum chamber walls. In order to model this behavior, it was assumed that the vacuum chamber walls are conical in shape, with the included angle equal to the angle with which the halo protons interact with the chamber wall. In the case of interest the angle was chosen to be $5 \mathrm{mrad}$. Thus a parallel proton beam interacting with this wall is equivalent to a 
halo proton beam which is diverging at an angle of $5 \mathrm{mrad}$, striking the cylindrical vacuum chamber wall. Coulomb scattering will cause a fraction of the protons to leak out and interact with down stream magnets. This approximation is not exactly equivalent to the actual case, since the actual halo will be traveling in the direction of the accelerator components (magnets etc.), as it leaves the vacuum chamber, which is not the case in the approximate model. However, in the approximate treatment the exposure of the components closest to the vacuum chamber wall might be higher than that of the components further away. The configuration to be considered below consists of a quadru-pole magnet connected to a $2 \mathrm{~m}$ length of vacuum chamber. Figs. 3 and 4 show the vacuum chamber quadru-pole magnet configuration. Fig. 3 shows the length of vacuum chamber and the attached magnet, with protons moving from left to right. Fig. 4 shows a cross section through the magnet. In addition an estimate was made of the activation due to irradiating a single piece of stainless steel. This is an idealized situation, since no piece of vacuum chamber is far from a component which can modify the surrounding radiation field. In both the above case involving a vacuum chamber the loss of halo protons was assumed to be 1 $\mathrm{W} / \mathrm{m}$ of chamber length. This loss rate translates into a proton current of $6.21504(9) \mathrm{p} / \mathrm{s}-\mathrm{m}$ for 180 days.

The linkage between LAHET/MCNP/ORIGEN-2S/MCNP calculations is shown on Fig.5. The geometric input for the LAHET and MCNP steps are identical, ensuring continuity. In addition, the gamma-ray source description in the last MCNP calculation varies with the volume being considered, thus accounting for spatial and spectral variations.

\subsection{Results}

The results to be presented below are given for the isolated vacuum chamber, vacuum chamber and magnet combination, the bare collimator, and several shielded collimator configurations. The first two shielded configurations have two thicknesses for the iron shield around the collimator. Additional configurations including a movable shield at the location of the highest potential dose were also considered. These results correspond to the time step immediately following machine shutdown. Finally, the variation of the dose with time following shutdown was also estimated.

\subsection{Isolated vacuum chamber}

The increase to the background dose in the tunnel due to the operation of the machine from losses to the vacuum chamber was estimated based on a loss rate of $1 \mathrm{~W} / \mathrm{m}$ of chamber length. At an energy of $1 \mathrm{GeV}$ and a chamber diameter of $20 \mathrm{~cm}$, a proton flux of $9.8915(5) \mathrm{p} / \mathrm{cm}^{2}-\mathrm{s}$ is implied. The above method was used to estimate the spallation product mass distribution for a 1 $\mathrm{cm}$ thick piece of stainless steel (type $304 ; 9 \% \mathrm{Ni}, 19 \% \mathrm{Cr}, 72 \% \mathrm{Fe}$ ) due to protons with an energy of $1 \mathrm{GeV}$. Following this step the time evolution of the spallation products was determined subjected to the neutron flux corresponding to the above proton flux for a time period of 180 days. The resulting gamma-ray source was then used in a transport calculation to determine the flux and hence the dose as a function of distance outside the vacuum chamber. The vacuum chamber was assumed to be a cylindrical structure with an ID of $20 \mathrm{~cm}$ and a wall 
thickness of $1 \mathrm{~cm}$. The dose conversion factors for gamma-rays are based on a American National Standard (ANSI/ANS-6.1.1-1997). The results are given in table 1.

Table 1 - Dose as function of distance from vacuum chamber

$\begin{array}{ll}\text { Distance }(\mathrm{cm}) & \text { Dose }(\mathrm{rem} / \mathrm{hr} .) \\ 10 \text { (surface) } & 11.1 \\ 41(\sim 1 \mathrm{ft} .) & 5.41(-2) \\ 110(1 \mathrm{~m}) & 6.64(-3)\end{array}$

It is seen that the dose drops off rapidly with distance, and is in the range of $\mathrm{mrem} / \mathrm{hr}$ beyond approximately $50 \mathrm{~cm}$.

\subsection{Vacuum chamber and associated quadru-pole magnet}

The case of a length of vacuum chamber connected to a ring component is a more likely scenario than the isolated piece of vacuum chamber considered above. The calculations were carried out using the geometric models shown in Figs. 3 and 4. Spallation product mass distributions were determined for a proton beam moving from left to right and interacting with the vacuum chamber wall at an angle of $5 \mathrm{mrad}$. The estimated proton current was based on a loss rate of $1 \mathrm{~W} / \mathrm{m}$ of vacuum chamber wall length with the machine operating for 180 days. Gamma-ray sources were determined for all the volumes of interest within the chamber walls and adjacent magnet structure. The dose was determined at various locations surrounding the vacuum chamber and magnet, resulting in a dose map.

The results of the calculation are shown in Fig. 3 for selected points around the chamber and magnet. It is seen that for the radial variation furthest from the magnet (extreme left hand side), the dose at $50 \mathrm{~cm}$ is equivalent to the dose at $41 \mathrm{~cm}$ for the isolated chamber case presented above. However, for the first $50 \mathrm{~cm}$ the radial variation for the isolated chamber case is much steeper (dropping off as $\sim \mathrm{r}^{2}$ ), while in the current case the drop off is milder (dropping off as r). The radial drop off increases as one approaches the magnet with a maximum drop off occurring right before of the magnet (drop off $\sim \mathrm{r}^{1.6}$ ). Beyond $50 \mathrm{~cm}$ the drop off is essentially inversely proportional to the radius. The maximum dose at $50 \mathrm{~cm}$ from the vacuum chamber surface is seen to occur approximately $1 \mathrm{~m}$ before the magnet and has a magnitude of approximately $110 \mathrm{mrad} / \mathrm{hr}$. This maximum is due to a combination of gamma-ray shine from the vacuum chamber and from the four magnet pole tips, since they are the exposed to the highest proton and neutron flux. Dose due to the remainder of the magnet structure is largely selfshielded, and thus the contribution to the tunnel dose is not as large.

\subsection{Inner collimator structure (unshielded and shielded)}

The inner collimator structure would be encountered when the collimator needs to be changed or repaired (although repairs are not necessarily practical in the usual sense), and would thus be removed from its shielded environment. Both unshielded and shielded $(10 \mathrm{~cm}$ inner shield) cases 
will be considered. The calculation was carried out using the collimator model shown in Fig. 2 . The spallation mass distribution was determined for a proton beam entering the front of the collimator and causing spallation reactions inside the collimator body. The shape of the halo beam was assumed to be cylindrical with its highest intensity closest to the inner radius $(2.5 \mathrm{~cm})$ and dropping off in a step-wise fashion to zero at the outer source radius $(4.5 \mathrm{~cm})$. This distribution was meant to mimic the actual distribution, although it is not clear what its shape would be, it will probably drop off with increasing radius. The estimate of the proton current was based on a loss fraction of 0.001 for a machine operating at $2 \mathrm{MW}$. This implies a current of 2.06(-6) Amps, and a proton current of 1.243(13) p/s. The operating period of the machine was assumed to be 180 days. A gamma-ray source was determined for all 27 internal volumes within the collimator. These are shown in the corresponding input file presented in the appendix. However, the sources corresponding to the water filled volumes were omitted, since it was assumed that the collimator would be drained at this stage, and thus a closer model of the real situation would be to have empty volumes in these cell. Thus, during the operating steps water was present in these volumes, and during the gamma-ray transport step the volumes were considered to be empty. This implies that the neutron flux would be softer during the 180 days of operation, affecting the reaction rates in a corresponding manner, and the empty cells will enhance the gamma-ray leakage during the maintenance phase. The dose was calculated at various locations outside the collimator resulting in a dose map.

The results of this calculation are shown in Fig. 6 for selected points outside the collimator. The "flash-light" effect, alluded to above, can be seen from the doses in the forward and backward directions along the beam lines. Doses in the backward direction are seen to be particularly high, since the primary proton beam enters at this point, and thus the gamma-rays source is more intense and is closer to the outside edge. In the forward direction the doses are lower by more than an order of magnitude. Figure 7 shows the variation of dose in the backward direction for various radial positions. This result shows the monotonic drop-off along the centerline, and as the radial position increases the dose starts off lower up against the collimator, increases to a maximum, and then decreases monotonically. The position of the maximum point increases in the axial direction with increasing radial position. This behavior is consistent with the "flashlight" effect. The same general dose vs. position map exists in the forward direction, except the values are all lower. Finally, it is seen that the doses along the proton beam-lines are quite high with a value of $13.5 \mathrm{rem} / \mathrm{hr}$ in the backward direction $2 \mathrm{~m}$ from the collimator face, and a value of $0.39 \mathrm{rem} / \mathrm{hr} 2 \mathrm{~m}$ in the forward direction. Remote handling and additional shielding will be required to move a collimator which has been in operation for an extended time ( 180 days).

The shielded configuration consists of the same collimator structure as used in the above calculation with the addition of a $10 \mathrm{~cm}$ thick iron shield enclosing the collimator. Appropriate openings are cut into the shield to allow vacuum chambers to pass through at either end. The dose for selected points around the collimator are shown on Fig. 8. The "flash-light" effect described above is evident, but the magnitudes are significantly lower than those determined for the unshielded case. Along the proton beam line, in the backward direction the dose is still approximately $5.5 \mathrm{rem} / \mathrm{hr}$ at a distance of $2 \mathrm{~m}$ (approximately half the value for the unshielded configuration), and a value of $0.2 \mathrm{rem} / \mathrm{hr} 2 \mathrm{~m}$ from the collimator face in the forward direction. Despite the addition of the inner shield it is seen that remote handling would be prudent. 
Finally, two configurations using the unshielded collimator were analyzed to determine the effectiveness of movable lead shielding. This movable shield took the form of a plug, which was inserted into the collimator tube in an effort to reduce the shine out of the tube. The second shield configuration included a lead slab, $18.5 \mathrm{~cm}$ thick, placed in front of the collimator in addition to the plug inserted in the collimator tube. Results for these calculations are shown in table 2 below. The results compare the unshielded collimator with the two shielded configurations in the backward direction (direction with highest dose). Radial distances are measured from the beam centerline and axial direction are measured from the collimator front face (negative sign implies backward direction).

Table 2 - Comparison of doses for unshielded collimator and including movable shields

\begin{tabular}{lllll}
\multicolumn{2}{c}{$\begin{array}{c}\text { Location } \\
\text { Number, Co-ordinates } \\
(\mathrm{r}, \mathrm{z})\end{array}$} & Unshielded & Plug only & $\begin{array}{c}\text { Dose (rem/hr) } \\
\text { Plug and slab shield }\end{array}$ \\
1 & $(0.0,0.0)$ & 9631.1 & 892.1 & $1.010(-2)$ \\
2 & $(0.0,-100.0)$ & 48.57 & 44.53 & $1.880(-3)$ \\
3 & $(0.0,-200.0)$ & 13.55 & 12.71 & $5.498(-4)$ \\
4 & $(47.5,0.0)$ & & & \\
5 & $(47.5,-100.0)$ & 49.24 & 20.86 & $1.494(-3)$ \\
6 & $(47.5,-200.0)$ & 13.82 & 36.28 & $8.804(-4)$ \\
& & & 12.01 & $5.066(-4)$ \\
7 & $(76.0,0.0)$ & 4.63 & 4.66 & $2.587(-2)$ \\
8 & $(76.0,-100.0)$ & 30.29 & 26.02 & $3.632(-4)$ \\
9 & $(76.0,-200.0)$ & 12.65 & 10.82 & $4.054(-4)$ \\
10 & $(175.0,0.0)$ & & & 0.80 \\
11 & $(175.0,-100.0)$ & 0.79 & 0.77 & 0.106 \\
12 & $(175.0,-200.0)$ & 7.24 & 6.23 & $2.808(-3)$ \\
& & 6.62 & 5.67 & \\
13 & $(275.0,0.0)$ & & & 0.29 \\
14 & $(275.0,-100.0)$ & 0.29 & 0.28 & $8.685(-2)$ \\
15 & $(275.0,-200.0)$ & 2.09 & 1.84 & $3.619(-2)$
\end{tabular}

The above results indicate that the addition of a lead plug in the collimator tube only reduces the dose at the point closest to the collimator along the beam centerline. All other dose values have essentially the same magnitude as the unshielded case. The addition of a movable shield in the form of a $18.5 \mathrm{~cm}$ thick lead slab before the collimator reduces the doses to significantly lower values. However, at radial distances beyond the lead shield the outer parts of the collimator shine through, and higher doses result. This analysis shows that it is possible to reduce the dose in the tunnel to acceptable values using appropriate shielding. It should be noted that the movable shields are heavy and will have to be moved remotely to avoid dose to operators. 


\subsection{Shielded collimator}

Under normal operating conditions the collimator will be shielded by $65 \mathrm{~cm}$ of iron in the axial directions (both forward and backwards), and by $20 \mathrm{~cm}$ of iron in the radial direction. This configuration is shown on Fig. 9. Also shown on Fig. 9 is the position of the moveable shield placed in the backward direction to aid in attenuating the relatively high doses along the beamline. The moveable shield could be used in the event of maintenance work with might be necessary on some component down stream of the collimator. A $18.5 \mathrm{~cm}$ thick layer of lead is assumed to be representative of the moveable shield. In order to compare the doses with and without the moveable shield present two calculations were carried out, and the dose determined at specific axial and radial positions. These locations are shown numerically on Fig. 9 together with the radial and axial distances. A comparison of the results is shown below in table 3 .

Table 3 - Doses for shielded collimator (standard configuration) with and without moveable shield

Location

Number, Co-ordinates

$$
(\mathrm{r}, \mathrm{z})
$$

$1 \quad(0.0,0.0)$

$2 \quad(0.0,-66.0)$

$3 \quad(0.0,-100.0)$

$4 \quad(0.0,-250.0)$

$5 \quad(47.5,-66.0)$

$6 \quad(47.5,-100.0)$

$7 \quad(47.5,-250.0)$

$8 \quad(175.0,-100.0)$

$9 \quad(325.0,-250.0)$

$10 \quad(0.0,136.0)$

$11 \quad(0.0,202.0)$

$12 \quad(0.0,236.0)$

$13 \quad(0.0,386.0)$

$14 \quad(47.5,202.0)$

$15 \quad(47.5,236.0)$

$16 \quad(47.5,336.0)$

$17 \quad(175.0,236.0)$

$18 \quad(325.0,386.0)$

$19 \quad(95.0,68.0)$

$20 \quad(175.0,68.0)$

$21 \quad(325.0,68.0)$
No moveable shield

9581.39

77.697

29.351

3.979

$2.168(-3)$

0.1314

1.067

$2.075(-3)$

$1.827(-3)$

105.038

1.4235

0.6905

0.1175

$3.245(-5)$

$1.097(-3)$

$7.935(-3)$

$5.742(-5)$

4.192(-5)

$8.005(-5)$

$1.378(-3)$

8.967(-4)
Dose (rem/hr)

With moveable shield

9586.34

$1.996(-3) *$

$1.502(-4)^{*}$

$4.455(-6)^{*}$

9.608(-6)*

$3.484(-4)^{*}$

$9.148(-5)^{*}$

106.505

1.4357

0.6905

0.1179

7.118(-6)

$1.097(-3)$

8.608(-3)

$5.742(-5)$

$5.081(-5)$

5.644(-5)

$1.378(-3)$

9.460(-4) 
* Behind moveable lead shield.

The above results indicate that the doses behind the shields are in the mrem/hr range. The only exception to this observation occurs in conical volumes centered on the entrance and exit to the collimator. In the upstream direction, along the beam axis, the dose starts at a maximum of $9581.39 \mathrm{rem} / \mathrm{hr}$, and decreases monotonically to a value of $4 \mathrm{rem} / \mathrm{hr}$ at a distance of $2.5 \mathrm{~m}$. By following the dose at a radius of $47.5 \mathrm{~cm}$ it is seem that it starts off at a low value $(2.2(-3)$ $\mathrm{rem} / \mathrm{hr}$ ) at the shield surface, and then increases to approximately $1.1 \mathrm{rem} / \mathrm{hr}$ at a distance of 2.5 $\mathrm{m}$. This behavior of the dose is consistent with the "flash-light" phenomenon described above. The same distribution occurs in the downstream direction, but the magnitudes are lower by more than an order of magnitude.

In order to reduce the dose in the "flash-light" zones to acceptable magnitudes a moveable shield can be placed in front of or behind the shield to augment the shielding thickness. It is seen from the above estimates that a shield consisting of $18.5 \mathrm{~cm}$ of lead reduces to magnitude of the dose along the beam centerline by approximately four orders of magnitude. These doses are thus acceptable for maintenance operations or for removal of the collimator.

\subsection{Time dependence of dose following machine shutdown}

The time dependence of the above dose levels can be estimated by determining the gamma-ray spectrum change with time as the various radioactive nuclides in the volumes of interest decay. This change in gamma-ray spectrum involves a reduction in the absolute magnitude of the source (photons/s), and a change in the energy distribution of the spectrum, since the various nuclides making up the source in a volume decay at different rates. It has been determined that for irradiated stainless steel the former effect (reduction in magnitude) is much more important than the latter effect (change in energy distribution). This suggests that for the collimator and vacuum chamber calculations described above the reduction in dose at any location can be estimated by reducing the dose determined at shutdown by the fractional change in gamma-ray intensity.

The reduction in gamma-ray intensity with time is shown in table 4.

Table 4 - Reduction in gamma-ray intensity (and dose) with time following shutdown

$\begin{array}{llllll}\text { Time since shutdown } & 0 & 1 \text { hour } & 1 \text { day } & 7 \text { days } & 30 \text { days } \\ \text { Fractional change } & 1.0 & & 0.3531 & 0.2224 & 0.1392\end{array}$

The above estimate indicates that after a month the dose has been reduced to approximately $15 \%$ of the value at shutdown. The drop off is relatively rapid in the first day and then slows down for the remaining time investigated. 


\subsection{Conclusions}

The following conclusions and recommendations can be made based on the above study:

1) The increase in background dose from to normal operations due to losses in the vacuum chamber wall are low but not negligible, and will increase with unintended beam losses,

2) Collimator shielding of the thickness suggested is necessary for acceptable dose levels in the tunnels adjacent to the collimators following machine shutdown.

3) A "flash-light" like beam of gamma-rays shines out of either end of the collimator tube, enhancing the possible dose in the upstream and downstream directions. This dose must be accounted for when scheduling maintenance and replacement of components in the vicinity of the collimators.

4) A moveable shield equivalent to approximately $18.5 \mathrm{~cm}$ of lead reduces the dose levels in the "flash-light" zone to acceptable levels.

\subsection{References}

1) R.E. Prael and H. Lichtenstein, "User Guide to LCS: The LAHET Code System", Los Alamos National Laboratory, Los Alamos, NM, LA-UR-89-3014 (1989).

2) MCNP-A General Monte Carlo N-Particle Transport Code Version 4A, J.F. Breismeister, ed., Los Alamos National Laboratory, Los Alamos, NM, LA-12625-M (1993).

3) W. B.Wilson, "Accelerator transmutation studies at Los Alamos with LAHET, MCNP, and CINDER-90", Los Alamos National Laboratory, Los Alamos, NM, LA-UR-93-3080 (1993)

4) A.G. Croff, "ORIGEN2 - A Revised and Updated Version of the Oak Ridge Isotope Generation and Depletion Code", Oak Ridge National Laboratory, Oak Ridge, TN, ORNL5621 (1980). 


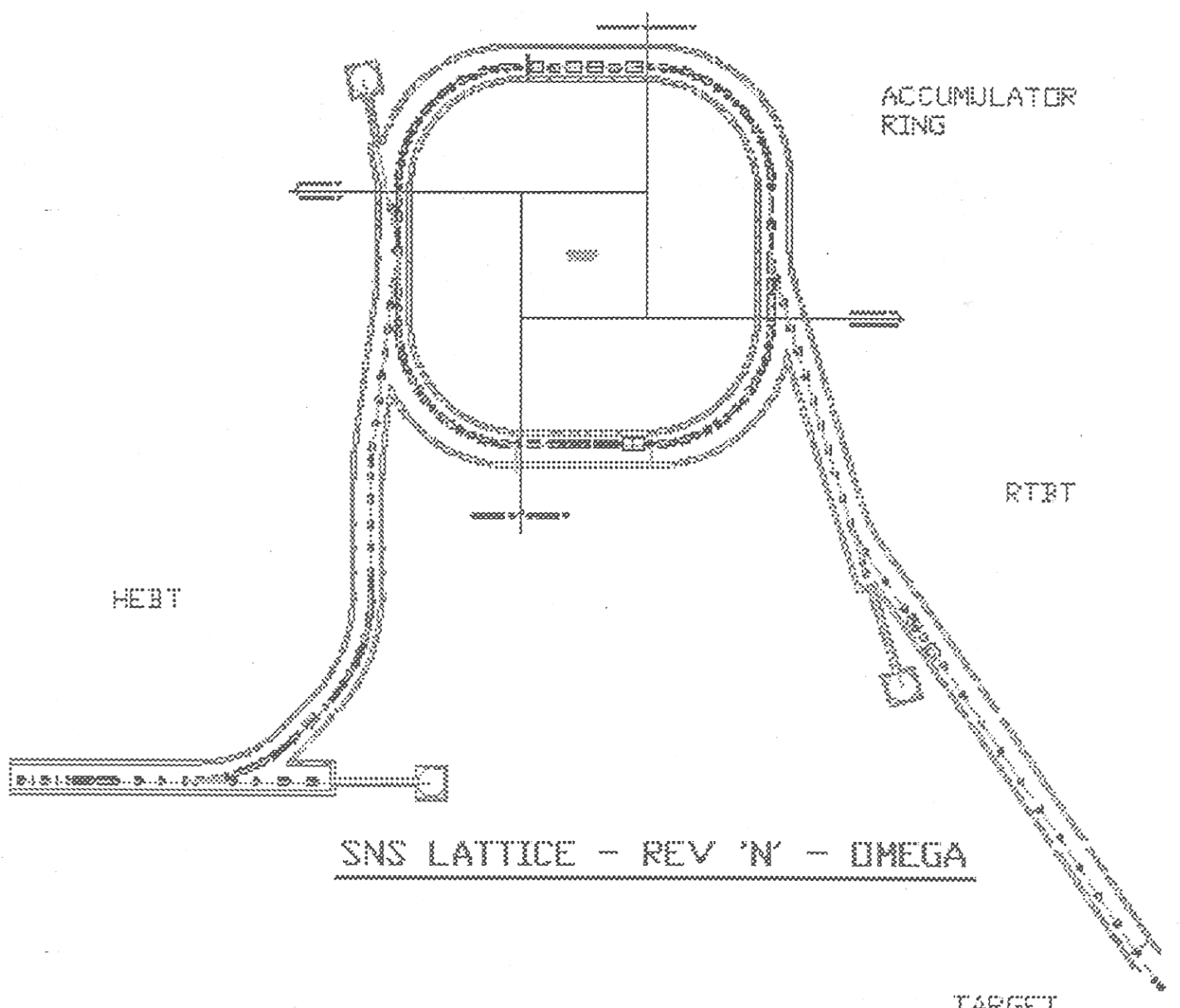

FAs $\{3\}$

Figure 1: SNS Omega Ring Configuration 


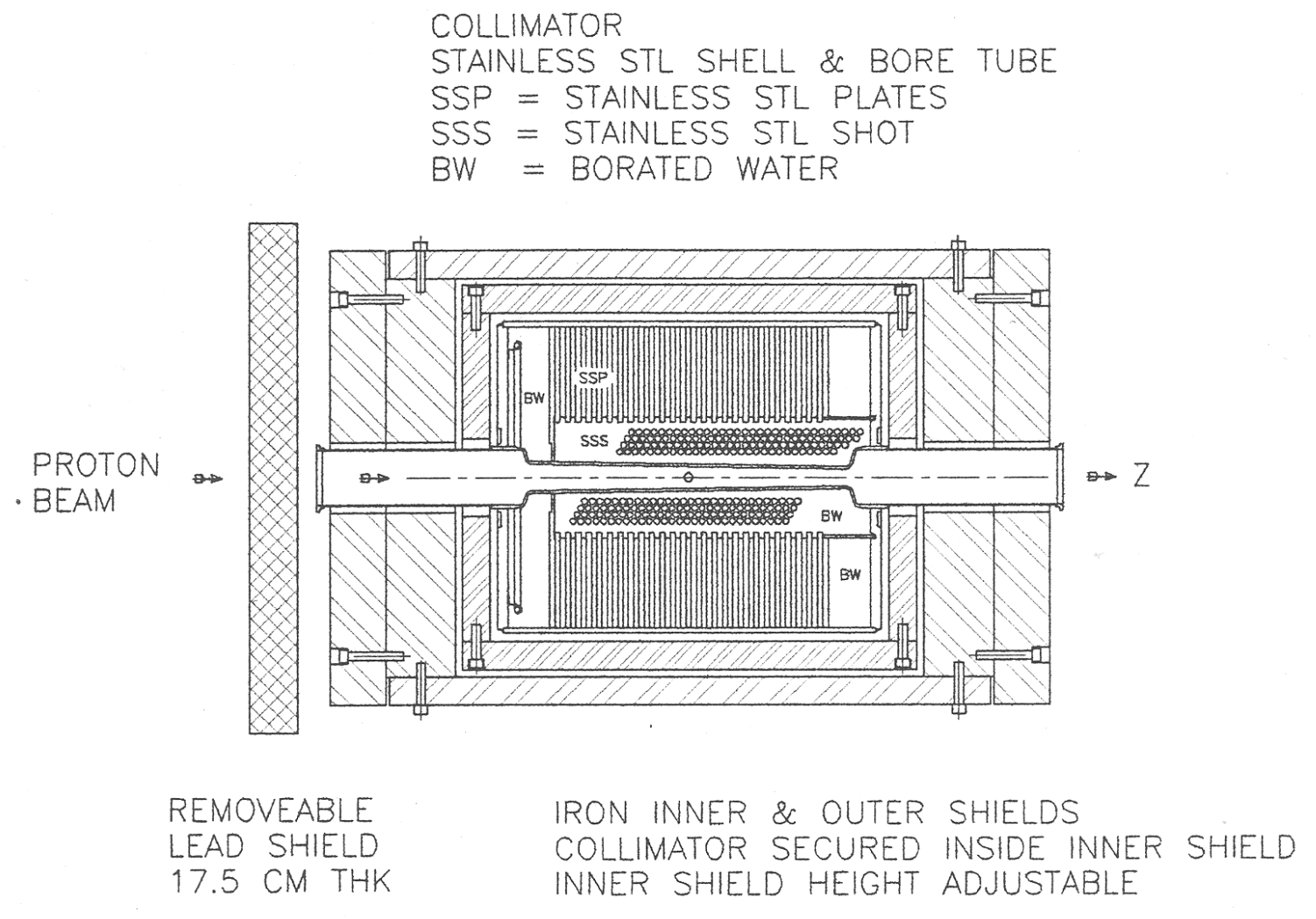

FIG 2 - SCHEMATIC OF COLLIMATOR COMPONENTS HORIZONTAL SECTION 


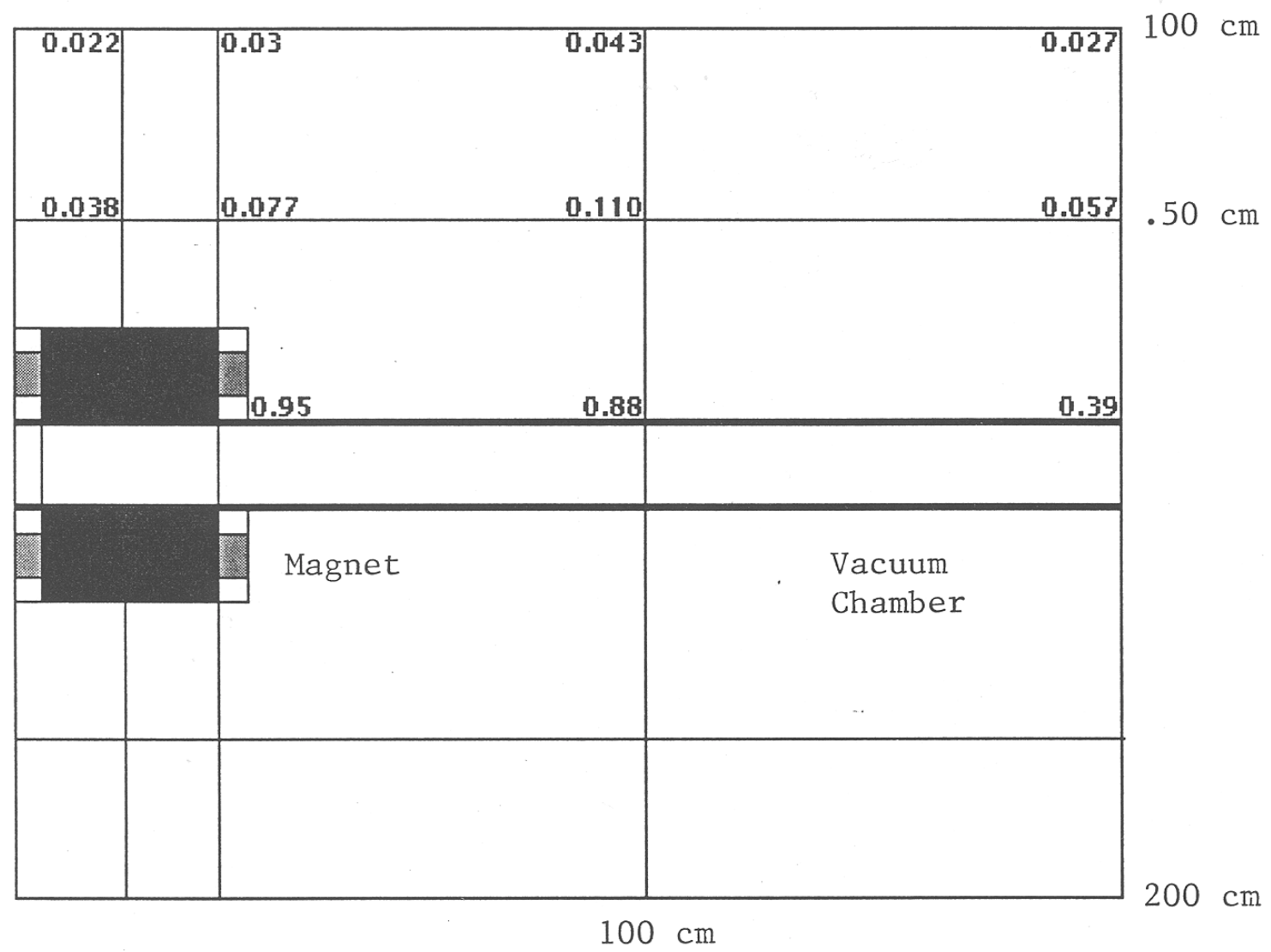

Figure 3: Quadru-pole Magnet with $2 \mathrm{~m}$ Vacuum Chamber (Dose in rem/hr) 
Iron

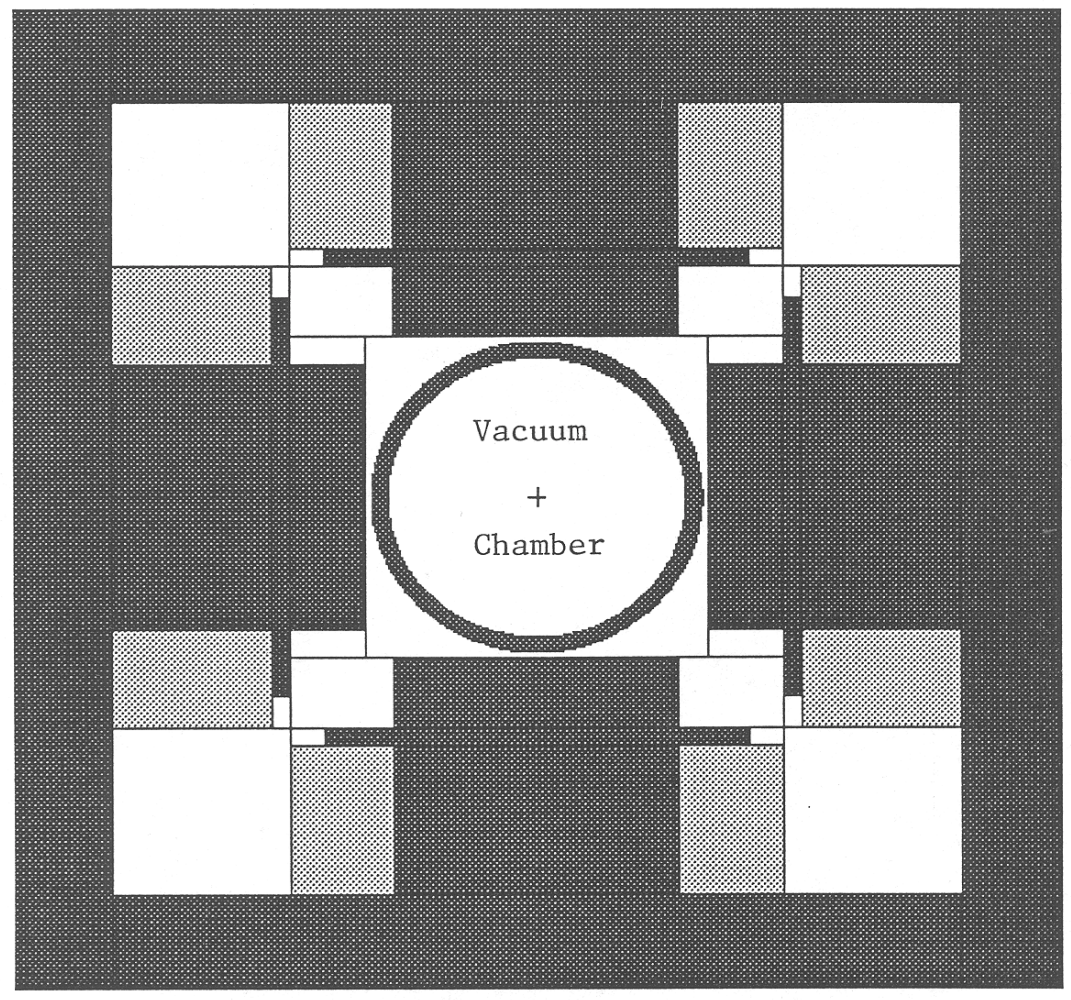

Copper Windings

Figure 4: Cross Section through Quadru-pole Magnet 


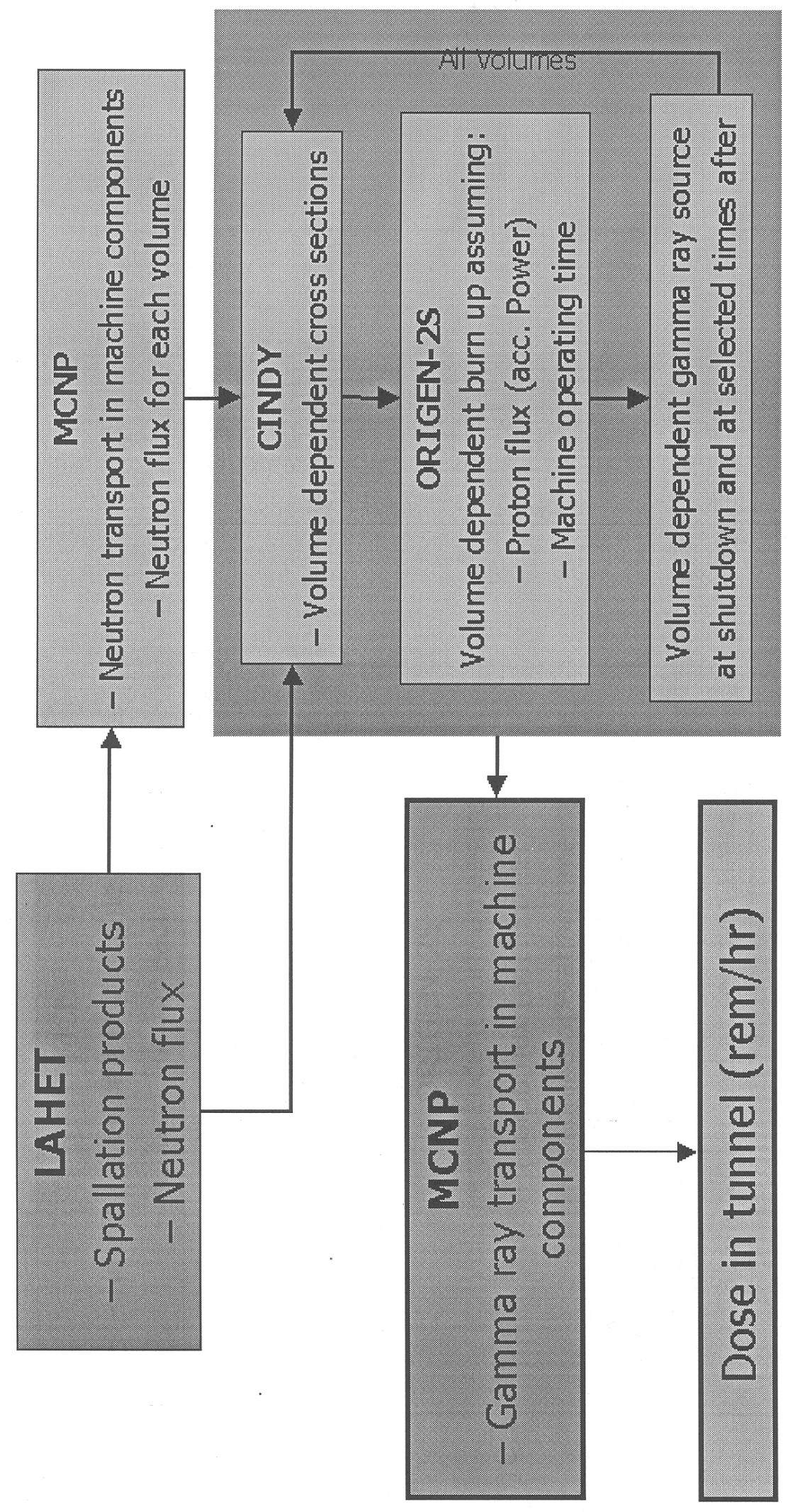




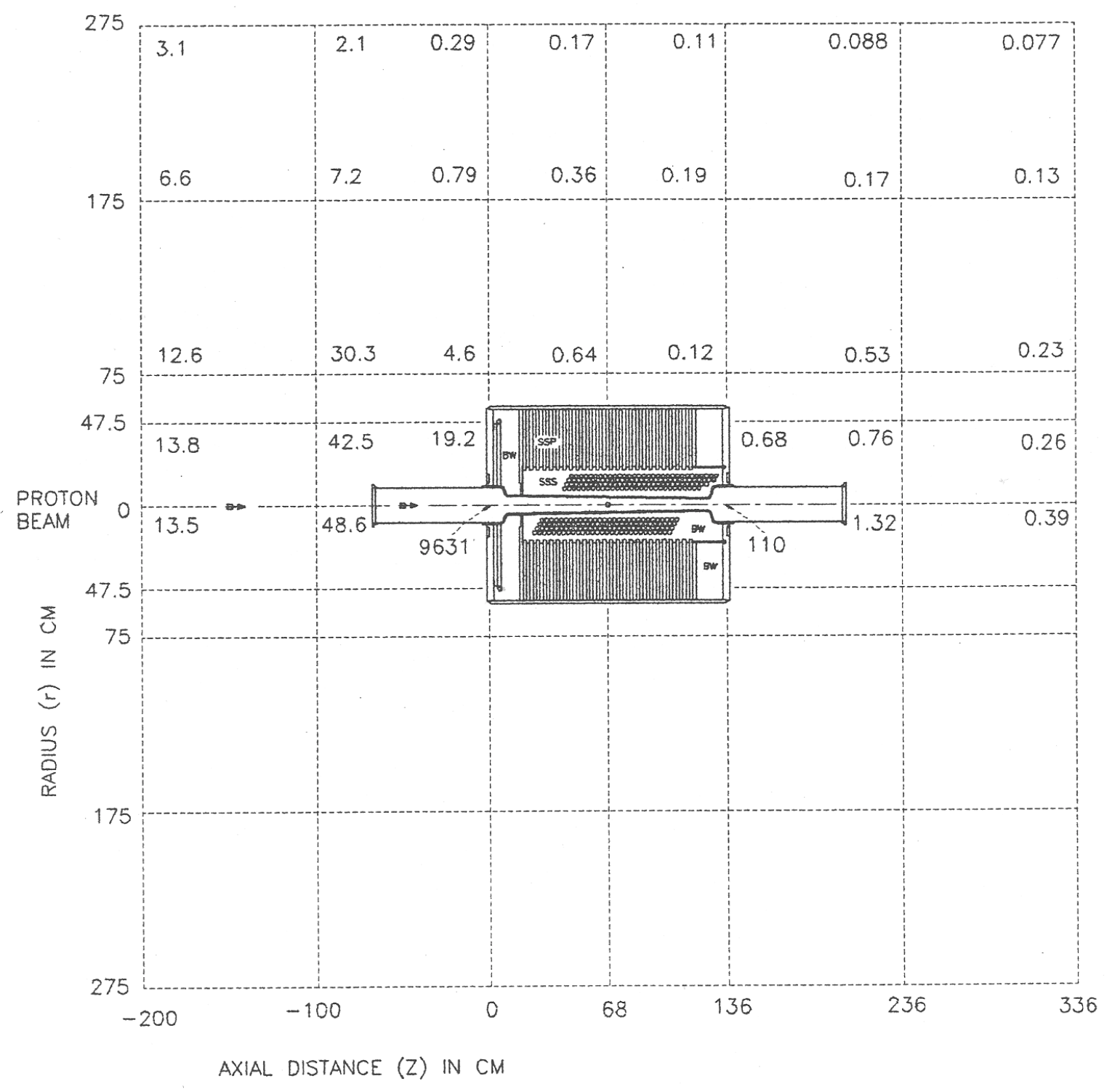

FIG 6 - DOSE IN REM/HOUR AROUND UNSHIELDED COLLIMATOR 
Figure 7-Dose vs distance and radius

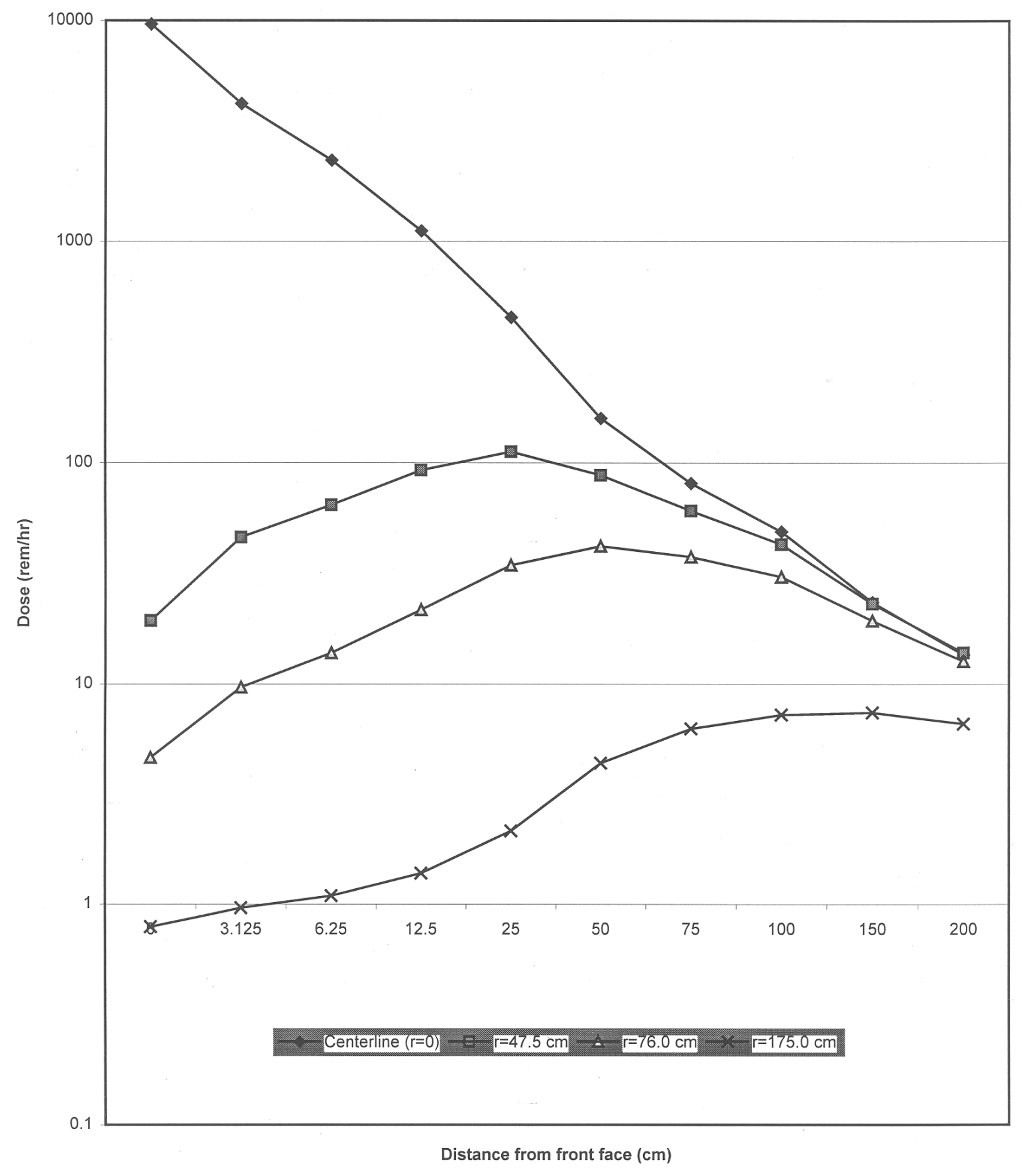




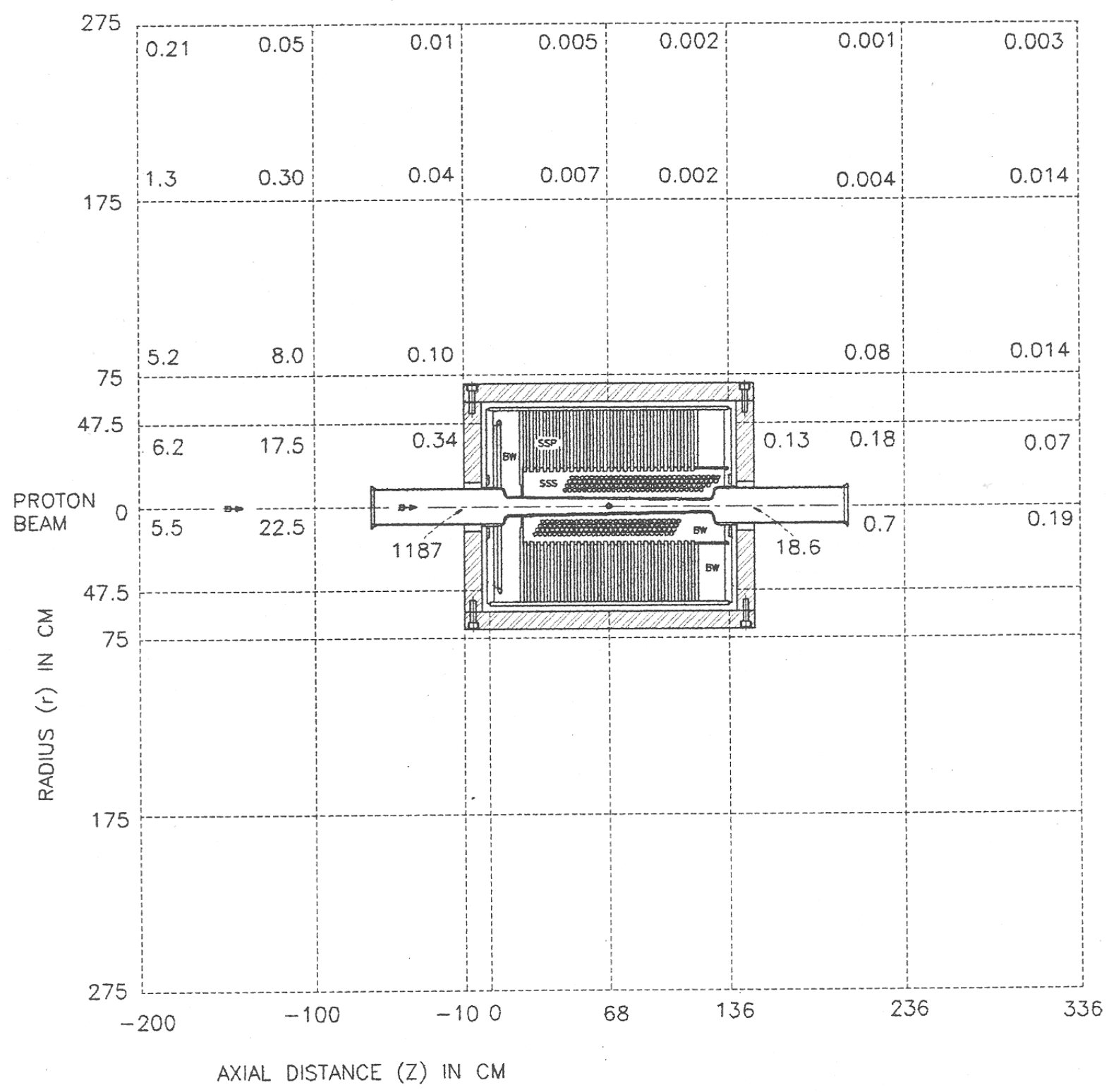

FIG 8 - DOSE IN REM/HOUR AROUND COLLIMATOR WITH 10 CM THICK INTERNAL SHIELD 


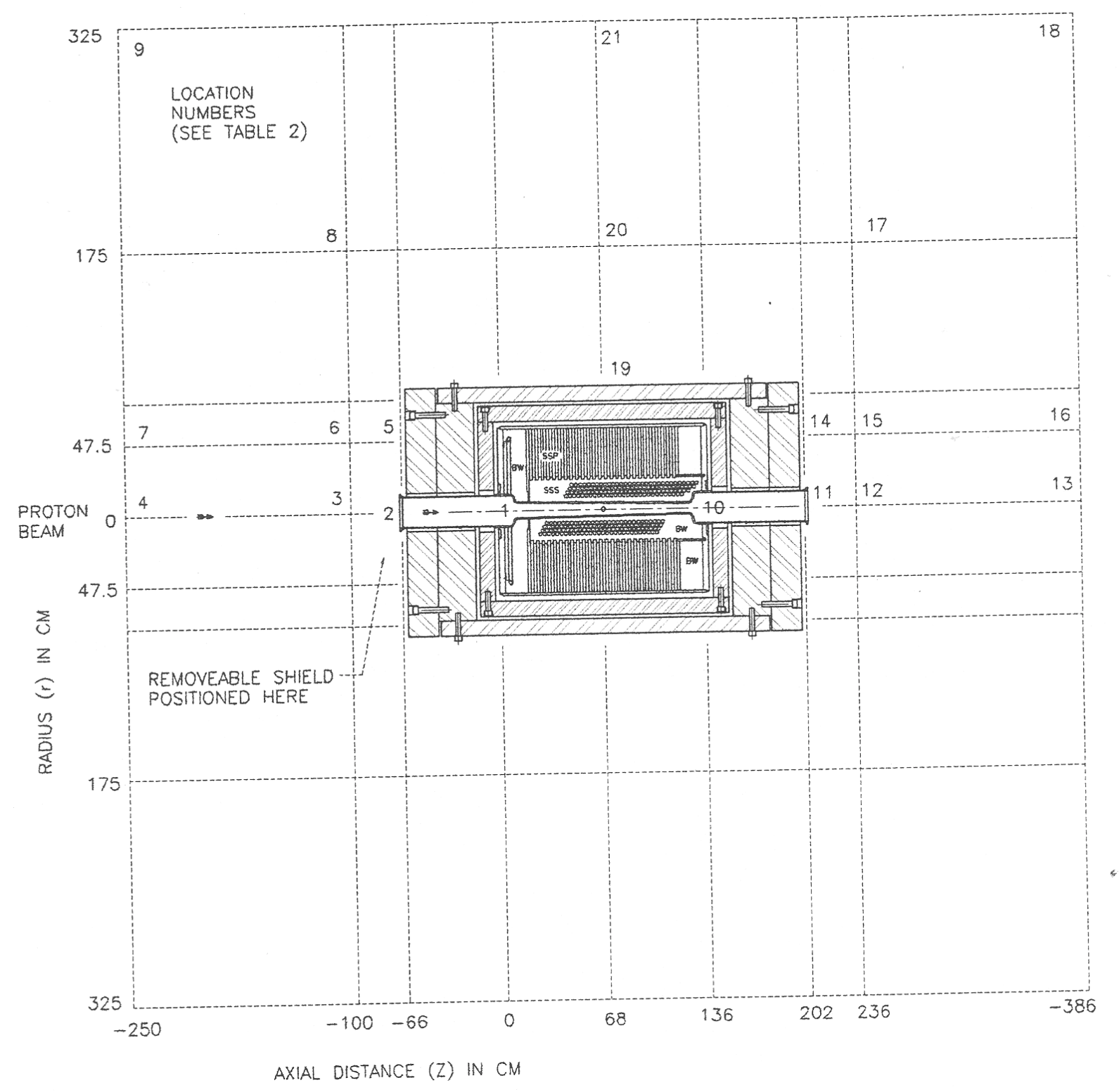

FIG 9 - LOCATION NUMBERS AROUND SHIELDED COLLIMATOR SEE TABLE 3 FOR DOSE IN REM/HOUR AT EACH LOCATION NUMBER 